\title{
Slow-release potassium and perforation of Meckel's diverticulum
}

\author{
G.T. Layer, R.H. Scott-Jupp, T.K. Maitra and S.G. Darke \\ Department of Surgery, Poole General Hospital, Dorset, UK.
}

\begin{abstract}
Summary: A case of peritonitis following perforation of gastric-type mucosa in a Meckel's diverticulum is described. At laparotomy a wax core of a combined diuretic - slow-release potassium preparation was found extruding through the perforation and a further pellet was found free in the peritoneal cavity. Intestinal perforation due to potassium-containing drugs is recognized and led to the development of enteric-coated and slow-release delivery systems. The association of perforation of Meckel's diverticulum with a slow-release potassium perforation has not previously been described.
\end{abstract}

\section{Introduction}

The administration of potassium supplements combined with diuretics protects against hypokalaemia, but it was recognized that the formulation, even when enteric-coated, was responsible for an increased incidence of gastrointestinal tract ulceration.' Slowrelease forms of potassium were developed to minimize this inherent risk and are now commonly prescribed. We report a case of ulceration and perforation of a Meckel's diverticulum following ingestion of such a preparation.

\section{Case report}

A 65 year old woman was admitted to hospital with a day's history of fever, diarrhoea and central abdominal pain. She had undergone appendicectomy 32 years previously and had recently been investigated for vague abdominal discomfort but no cause had been identified. Six months before admission she had begun taking a combined diuretic-slow-release potassium preparation (bumetanide $500 \mathrm{mg}$ and potassium chloride slow-release $573 \mathrm{mg}$, Burinex-K, Leo Laboratories Ltd.) twice daily to reduce mild ankle oedema. She also required sustained-release indomethacin $75 \mathrm{mg}$ capsules (Indocid-R, Thomas Morson Pharmaceuticals) at night for symptomatic cervical spondylosis. Soon after admission she

Correspondence and present address: G.T. Layer, M.A., F.R.C.S., Department of Surgery, St Thomas' Hospital, London SE1 7EH, UK.

Accepted: 10 September 1986 developed signs of peritonitis. Investigations revealed a haemoglobin of $10.9 \mathrm{~g} / \mathrm{dl}$, a toxic neutrophilia and free intra-peritoneal gas. Laparotomy confirmed peritonitis due to an inflamed and perforated Meckel's diverticulum. Extruding through the perforation was a soft brown diamond shaped pellet (Figure 1). There was a further similar pellet lying free in the peritoneal cavity. These were later identified as the wax cores of the diuretic - slow-release potassium tablets. A Meckel's diverticulectomy followed by peritoneal lavage was performed. The patient made an uneventful recovery.

Histology of the excised diverticulum confirmed gangrene and perforation in areas of gastric-type mucosa.

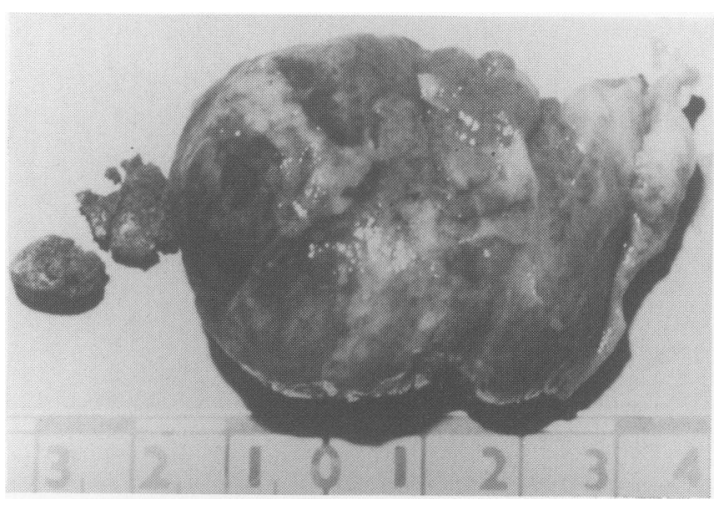

Figure 1 The wax core of a Burinex-K tablet extruding from the perforated Meckel's diverticulum.

(C) The Fellowship of Postgraduate Medicine, 1987 


\section{Discussion}

Perforation of the small bowel is reported in association with many bizarre foreign bodies. ${ }^{2}$ There is a tendency for these to lodge in blind pouches and ulceration of a Meckel's diverticulum has been described due to an enteric-coated potassium chloride tablet. $^{3}$

Ulceration, stenosis and perforation of the gastrointestinal tract is recognized following both potassiumand indomethacin-containing preparations. That due to indomethacin may be caused by potassium bicarbonate in the formulation. ${ }^{4}$ Despite the introduction of slow-release drugs there have been reports of mucosal ulceration, ${ }^{5}$ apparently associated with some degree of stasis whereby more potassium was released adjacent to an area of mucosa than would normally occur.

In our unique case the patient was taking a preparation consisting of an outer shell of diuretic designed to dissolve within 30 minutes and an inner core of potassium chloride in an inert wax base intended to be

\section{References}

1. Davies, D.R. \& Brightmore, T. Idiopathic and druginduced ulceration of the small intestine. Br J Surg 1970, 57: 134-139.

2. Lumsden, A.B. \& Dixon, J.M. Tomato skins penetrating the small bowel. Br J Surg 1984, 71: 648.

3. Hasker, W. \& McCaffret, J. Ulceration of a Meckel's diverticulum due to a potassium chloride tablet. Med $J$ Aust 1972, 2: 261-262.

4. Day, T.K. Intestinal perforation associated with osmotic slow release indomethacin capsules. $\mathrm{Br} \mathrm{Med} J$ 1983, 287: 1671-1672. released over 6 hours to avoid high local concentrations. Thus, when the tablet reaches the distal ileum $\frac{3}{8}$ the diuretic coat has been absorbed and the foreign $\varrho$ body core still containing potassium can become sequestered into a blind diverticulum, causing ulceration and perforation.

Areas of heterotopic gastric mucosa often present in Meckel's diverticulae, and identified in this case, are particularly susceptible to potassium-induced ulceration. However, this complication must be rare since the accepted incidence of the diverticulum is only $2 \%$. The risks of oral potassium therapy must be further $\overrightarrow{0}$ considered and other methods developed to ensure $\vec{A}$ safer delivery of the drug, such as the Continus System (Napp Laboratories Ltd). ${ }^{6}$

\section{Acknowledgements}

The authors thank Dr J.S. Howell, Consultant Pathologist, Poole General Hospital and Dr D.R. Davies, Senior Lecturer iv in Pathology, St Thomas' Hospital for their assistance in the $\overrightarrow{-}$ preparation of this report.

5. Farquharson-Roberts, M.A., Giddings, A.E.B. $\& \overrightarrow{0}$ Nunn, A.J. Perforation of small bowel due to slow release potassium chloride (Slow-K). Br Med J 1975, 留; 206.

6. McLoughlin, J.C. Effects on upper gastrointestinaี mucosa of three delivery systems of potassium as sup- $\bar{\partial}$ plement to frusemide administration. J Roy Soc Med 1985, 78: $459-462$. 